ALPHA Nº 28 Julio 2009 (169-180)

ISSN 0716-4254

http://alpha.ulagos.cl

\title{
EL PAR SIMULACIÓN DISIMULACIÓN Y EL ARTE DE
} SABER VIVIR

The pair dissimulation simulation and art of good living

Boris Eremiev Toro*

Nonne dissimulavi? Nonne silui? Nonne quievi? et venit super me indignatio.

Job. 3, 26.

Ecce ego mitto vos sicut oves in medio luporum; estote prudentes sicut serpentes et simplices sicut columbae. Mt. 10, 16.

Resumen

La intención del presente trabajo es mostrar cómo, durante el Barroco, las figuras retóricas de la dissimulatio y la simulatio, pasan a ser estrategias para enfrentar las dificultades de la vida, según se ilustra en los tratados de Baltazar Gracián y Torcuato Accetto.

Palabras clave: Ironía, Barroco, retórica, moral.

Abstract

The purpose of the following article is to show the changes suffered by the notions of dissimulatio and simulatio during the Baroque period. Specifically, my intention is to illustrate, through the works of Gracian and Accetto, how these rhetorical figures become ways of confronting life.

Key words: Irony, Baroque, rhetoric, morality.

\section{INTRODUCCIÓN: LA RETÓRICA Y EL VIVIR BIEN}

Es mi propósito tratar de un modo peculiar la relación entre retórica y ética. Digo peculiar, porque no pretendo referirme al problema de los límites que se establecen entre ambas disciplinas, a saber, si la retórica debe o no sujetarse a la ética; si de algún modo ésta la constriñe o si, al contrario, la retórica devora a todas las ramas de la filosofía y, por ende, a la misma ética. Por supuesto, no es que estos asuntos se consideren zanjados, o irrelevantes. Más bien, mi intención es investigar sobre un tema que, en mi experiencia, me ha parecido dejado de lado, como es la transformación de la retórica en ética. Dicho así, claro está, parece una cosa peregrina, pues ¿Cómo una disciplina cuyo fin es la persuasión o encontrar los mejores medios para hacerlo va a 


\section{Boris Eremiev Toro}

versar sobre lo bueno y lo malo? No tengo una respuesta a esta pregunta. Pero no es mi intención, siquiera, tratar de responderla, pues mi objetivo es otro. Lo que quiero es investigar cómo algunos mecanismos, o mejor dicho, figuras retóricas, se deslizaron del campo de la retórica al ámbito de la moral, pasando a formar parte de las estrategias correspondientes al ars bene vivendi. Para hacerlo pretendo trabajar sobre la simulación y la disimulación. Siguiendo su aparición en determinados escritos, me esforzaré en bosquejar una pequeña historia de ellas, para ilustrar cómo se produjo este desplazamiento y qué consecuencias implica. Partiré utilizando la definición que da Accetto de ellas, para luego mostrar los comentarios de Aristóteles y Cicerón ${ }^{1}$ al respecto. Después, utilizaré el Oráculo Manual de Baltasar Gracián y el Tratado sobre la disimulación honesta de Torquatto Accetto como casos paradigmáticos del uso de estas figuras dentro del campo de la vida práctica. La elección de estos textos no es gratuita. En cuanto a la primera obra mencionada, la elijo porque, a mi juicio, es un decantado del pensamiento del P. Baltasar Gracián y una guía moral que enseña a desenvolverse en el mundo en virtud de la apariencia. En cuanto al de Accetto apenas requiere justificación, el propio texto está consagrado a defender la disimulación como un modo honesto de vivir y enfrentar a nuestros semejantes.

\section{JACTANCIA, IRONÍA Y EL PAR DI/SIMULACIÓN}

En un primer momento Accetto nos da la clave para comprender el par disimulación/simulación. En su Tratado sobre la disimulación honesta las define del siguiente modo: "La disimulación es la acción de no hacer ver las cosas como son. Se simula lo que no es, se disimula lo que es".

Así, la simulación y la disimulación se definen como técnicas de la apariencia, ya sea como arte del encubrimiento, ya sea como una forma de fingir. Sin embargo, su origen poco tiene que ver con el uso barroco ${ }^{3}$ de estas

\footnotetext{
${ }^{1}$ Las citas a Aristóteles corresponden a la edición de Bekker. Las referencias a Cicerón corresponden al sistema de citas correspondiente a los textos clásicos (Autor, sigla de la obra, libro, capítulo, verso, así por ejemplo, Cic. Cat. I.1.1).

2 Torcuato Accetto. La disimulación honesta. Buenos Aires: El cuenco de plata, 2005:109. Citaremos por esta edición.

${ }^{3}$ Este movimiento del siglo XVII suele comprenderse por oposición al Renacimiento, como lo hizo Wölfflin. Según Hatzfeld, Wölfflin “...opone el Barroco al estilo que fue su inmediato predecesor en Italia, el Renacimiento, y presenta al Barroco como una visión pictórica en contraste con la visión lineal del renacimiento. Oponía también la profundidad a lo meramente superficial; la unidad a la multiplicidad, la complejidad a la densidad; la oscuridad a la claridad. El Barroco es el estilo del punto de vista pictórico con perspectiva y profundidad, que somete la multiplicidad de sus elementos a una idea central, con una visión sin límites y una relativa oscuridad que evita los detalles y los perfiles agudos, siendo al mismo tiempo un estilo que, en lugar de revelar su arte lo esconde” (1966:15).
} 
figuras. En un primer momento dissimulatio y simulatio se vincularon a los términos griegos aladzoneia (jactancia) y eironeia (ironía) los que fueron tratados por Aristóteles en su Ética nicomaquea. De este modo, puede ser útil tener a la vista, de manera sumaria, las consideraciones que Aristóteles nos ofrece en esa ética a Nicómaco respecto de estos términos. ${ }^{4}$ El Estagirita busca establecer el término medio entre estos extremos (la jactancia y la ironía). Lo primero que nos dice es que este término medio carece de nombre. Sea como sea, se trata de evaluar a los hombres en conformidad a que sean verdaderos o falsos. El jactancioso es aquel que se atribuye lo que le da la gloria; en cambio, el irónico niega o le resta importancia a lo que le pertenece, mientras que el sincero reconoce las cualidades que posee sin agregar o quitar ninguna. Aristóteles considera reprensibles ambos extremos, pero sobre todo el jactancioso. Aunque nos dice, moderando de este modo su crítica, que es más bien vano que malo. El irónico, a su juicio, es más agradable, pues más que afán de ganancia busca rehuir la ostentación (EN 1127a-1127b). Tenemos que observar que esta correspondencia va a mostrar un desplazamiento. Del tratamiento ético al cual se vieron sujetas en Aristóteles pasarán, también, a formar parte de la retórica. Esto lo podemos observar, por ejemplo, en el De oratore de Cicerón. En efecto, en este texto la dissimulatio parece tratada dentro del campo del humor como medio de persuasión (De orat. II, 216290). En concreto, después de establecer las especies del humor, ${ }^{5}$ se sostiene

\footnotetext{
${ }^{4}$ Pavlovskis dedica un artículo al tema de la ironía como carácter. Sus fuentes de reflexión son Aristóteles y Horacio, debido a que en el período que transcurre entre ellos no hay, a juicio de Pavlovskis, un tratamiento que valga la pena o que no sea desdeñoso en relación con el hombre irónico. Pavlovskis puntualiza que la etimología de eiron es oscura y que parece haber sido vinculada a un uso despectivo. Posiblemente, aclara, la aplicación de este término a Sócrates podría haberle ganado respeto, aunque el propio Platón no es del todo amable con la ironía (Rep. 1, 337a). No obstante, es en Aristóteles donde podemos encontrar una consideración más positiva de este asunto. En La Retórica entrega una imagen negativa de irónico como de alguien que puede irritar a sus oyentes (Rhet, 1379b31-32) o que es poco confiable (2006: 1382b19-22), para luego considerar que es una forma de humor apropiada a los caballeros (2006:1419b5-9). En Physiognomica nos da una descripción física del hombre irónico (2006: 808a27-29). Además, están los pasajes correspondientes en la Ética a Eudemo (1233b381234a3). Pavlovskis considera que estos pasajes implican una dificultad, a saber, reconciliar el carácter magnánimo (veraz) con afirmaciones positivas acerca de la ironía. Por medio de la figura de Sócrates el intérprete esboza una solución, a saber, la ironía es conforme a la grandeza de alma, cuando es usada con fines pedagógicos (Pavlovskis, 1968:22-41).

${ }^{5}$ El diálogo parte con la invitación de Marco Antonio a que César, debido a su experticia en el humor, se explaye sobre el asunto. Al principio Cicerón nos lo muestra reacio, pues la primera afirmación es que el humor no está sujeto a teoría. No obstante, César complace a sus auditores y comienza estableciendo la diferencia entre el humor que impregna todo el discurso (cavillatio) y el que es breve y punzante (dicacitas). Luego, puntualiza sobre la utilidad para el orador que posee el humor, a saber, éste es una excelente arma refutatoria. Según César, hay una serie de cuestiones que deben responderse: i) en qué consiste la risa, ii) de dónde proviene,
} 


\section{Boris Eremiev Toro}

que la disimulación/ironía ${ }^{6}$ es una de las formas elegantes en que se puede lograr el humor basado en la situación. ${ }^{7}$ Sin embargo, no dejan de ser objeto de juicio moral, pues el propio Cicerón las considera brevemente en el Libro tercero del De officis donde trata de lo útil y lo honesto y, especialmente, de los medios de resolver un posible conflicto entre ambos. Dentro de este campo establece una norma general, según la cual nunca debemos engañarnos por la apariencia de una falsa utilidad. Así, llega al principio estoico de que lo bueno y lo útil se identifican. Ahora bien, basándose en el principio anterior, en De officis III:15-61, Cicerón se pronuncia sobre la disimulación y la simulación, condenándolas cuando son parte de la vida (Ex omni vita simulatio dissimulatioque tollenda est). Si consideramos estos desarrollos, el uso y la alabanza de, al menos, la disimulación parece paradójico ¿Cómo es que de una reprobación del uso práctico de este par llegó a ser objeto de tratados laudatorios en el s. XVII respecto a su uso? Una primera repuesta la podemos obtener con la ayuda de Remo Bodei para quien el disimulo es

una forma de resistencia racional y creativa a la opresión de un poder que comenzaba a infiltrarse directamente en las conciencias, también para llenar el vacío de hegemonía interior dejado por los cismas

iii) es propio del orador querer provocar risas, iv) hasta qué punto y v) los tipos de lo risible. Después de resolver estos temas, se distinguen dos medios que provocan la risa: por situación y por palabra que se reconoce porque toda su gracia estriba en los términos en que se expresa el orador, dejando de producir su efecto, si las palabras son cambiadas. En la risa provocada por situación se mantiene el efecto, sin importar el hecho de que se cambien las palabras. Pertenecen al primer tipo: lo ambiguo, lo inesperado, la paranomasia, alegoría, etc.; al segundo, la narración, las imágenes, el sobrentendido, aparentar no entender y la ironía (dissimulatio).

${ }^{6}$ Dissimulatio no es la única manera en que Cicerón se refiere a la eironeia. También se vinculan a ella los siguientes términos: ironia (infrecuente), simulator, dissimulator; y frases como: inversio verborum, invertere verba y de se detrahere (Canter, 1936:457, $\mathrm{N}^{\circ} 1$ ).

${ }^{7}$ El uso de la ironía por parte del propio Cicerón en sus discursos, parece ser un poco más complejo de lo que el propio orador nos cuenta. Según H. V. Canter, podemos apreciar una diferencia entre la teoría de la ironía, que tiende a concebirla dentro del ámbito de lo gracioso, y el uso real de ella por parte de Cicerón. En este último caso, la ironía puede ir desde una ironía ligera hasta el sarcasmo. De este uso, Canter concluye que la ironía llega a ser un hábito de pensamiento, un instrumento de defensa y de ataque. De ahí, Canter pasa a establecer las siguientes especies del uso de la ironía en Cicerón: i) el orador pretende no decir una cosa, pero, no obstante, la dice, ii) el orador finge pasar algo por alto, pero no lo hace, iii) se le da énfasis a una cosa por su omisión, iv) cuando el orador finge no saber qué hacer o qué decir, v) concesiones irónicas, vi) se finge falta de habilidad o ignorancia, y vii) sarcasmo. Canter también hace notar, en relación con la frecuencia y extensión de los pasajes que suelen contener una ironía, que éstos son normalmente breves y que se presenta sin grandes diferencias entre los distintos tipos de géneros oratorios, aunque el judicial presenta pasajes de mayor extensión. En cuanto a los estilos, es más frecuente en el genus tenue y casi desaparece en el genus grande. Al interior del propio discurso, es más frecuente en la argumentatio que en el exordio o en la peroratio (Canter, 1936). 


\section{El par simulación disimulación y el arte de saber vivir}

teológicos y por las guerras de religión que desgarraban a Europa (1995:139).

El disimulo es entonces, según el comentarista italiano, una estrategia de supervivencia y su uso una necesidad ante una forma de poder penetrante y totalitaria. ${ }^{8}$ Así, se distinguen en el período Barroco dos usos, a saber, un uso privado e ilegítimo, y otro público y honesto. Además, la simulación se transforma en un arte que tiene como uno de sus resultados el conocimiento y el dominio de sí. Por último, el disimulo ante sí mismo se convierte en un medio de alcanzar una forma efímera de felicidad, la cual - a veces- es la única a la que se puede aspirar. De este modo, la transformación a la cual nos referíamos ya no es objeto de sorpresa; pues, la simulación y la disimulación se convierten en parte de una nueva forma de prudencia, la cual no se define tanto por la administración de los placeres o por la adquisición del justo medio, sino por la cautela. ${ }^{9}$ Veamos cómo estos temas son desarrollados en Gracián y Accetto. ${ }^{10}$

\section{GRACIÁN Y ACCETTO: SIMULACIÓN Y PRUDENCIA}

Para Gracián, el mundo y el hombre se han convertido en algo complejo cuyo entendimiento excede a la tradicional forma de comprender, ${ }^{11}$ de modo que frente a ello, el hombre debe desarrollar un arte capaz tanto de distinguir

\footnotetext{
${ }^{8}$ En efecto, el periodo mencionado puede interpretarse en términos políticos, como una cultura de conservación del estado monárquico. En concreto, el arte exagerado del Barroco sería una forma de escapismo y de distracción de los miembros que no estaban conformes con la disposición económica y social del Estado. En tanto, los políticos se preocupaban por mantener la situación, estableciendo cotos a la enseñanza, esto es, al principal medio de movilidad social de la época. Desde esa perspectiva, el saber y, más aún, la ostentación del saber se volvía una arma de doble filo: frente al poderoso era un riesgo mostrar lo que se sabe, pero la mera ignorancia suponía una condena al estado social en que se nacía (Maravall, 1975:274-275).

9 Todo esto es un reflejo de la situación del hombre barroco. En efecto, la confianza y transparencia del Renacimiento, cede paso en el Barroco a la desconfianza y a la cifra (Hatzfeld, 1966:120-130).

${ }^{10}$ La elección de estas figuras responde a que son representantes de los polos geográficopolíticos del Barroco. Por un lado, Accetto corresponde a quien vive sujeto al orden político de las ciudades Estados (en concreto Nápoles) de la actual Italia; Gracián, es el representante del hombre de letras que vive subordinado al Imperio Español. Es decir, tenemos a dos figuras que utilizan estrategias de supervivencia, semejantes o iguales, pero en contextos políticos que si bien pertenecen al Barroco, muestran profundas diferencias en lo relativo a su ordenamiento político y económico.

11 "Más se requiere hoy para un sabio que antiguamente para siete; y más es menester para tratar con un solo hombre en estos tiempos que con todo un pueblo en los pasados”. Baltazar Gracián. Oráculo Manual y arte de la prudencia. Barcelona: Debate, 2002:I. Citaremos por esta edición, indicándose los aforismos con números romanos.
} 


\section{Boris Eremiev Toro}

las apariencias como de fingirlas y ocultarlas. ${ }^{12}$ Se trata de moverse en un terreno peligroso y falaz ${ }^{13}$ donde la facultad de enfrentar lo que nos rodea no es capaz de decantar del todo en un sistema de reglas o de preceptos uniformes. ${ }^{14}$ De este modo, las sentencias que nos ofrece el jesuita suelen no sólo no encajar, sino que acostumbran contradecirse, resultando muy difícil subordinar sus máximas a un principio común. ${ }^{15}$ Dentro de esta complejidad, la relación con los otros hombres es de desconfianza, ya que cada semejante es un posible rival. Lo anterior explica, al menos hasta cierto punto, la actitud recelosa de Gracián frente a la amistad; por un lado, la amistad es cosa necesaria, pues el trato con aquellos que poseen virtudes es una escuela de enseñanza, redundando su contacto en nuestro provecho; por otro, el amigo es un potencial enemigo y, quizás, el más peligroso de todos. Con lo anterior, poco a poco, se nos va revelando el mundo social al cual nos remite la obra de Gracián: se trata de una región de simulacros y apariencias donde las pasiones son el punto de fuga de nuestro ánimo; por lo mismo, su expresión debe atemperarse a fin de que nuestra intimidad permanezca intacta frente a los embates de lo social (2002:XCVIII). En consecuencia, una de las normas de este obrar es el dominio de las pasiones, ejercicio que tiene por fin, precisamente, vedar la manifestación de nuestros sentimientos; la voluntad, de este modo, queda "cifrada”. Por un lado, control de las propias pasiones; por el otro, la conveniencia de controlar las ajenas. Así, la capacidad de torcer la voluntad ajena, captando sus deseos para volcarlos en nuestro beneficio es

\footnotetext{
12 “Arte era de artes saber discurrir: ya no basta, menester es adivinar, y más en desengaños. No puede ser entendido el que no fuere buen entendedor. Hay zahoríes del corazón y linces de las intenciones. Las verdades que más nos importan vienen siempre a medio decir; recíbanse del atento a todo entender: en lo favorable, tirante la rienda a la credulidad; en lo odioso, picarla” (2002:XXV).

13 "Milicia es la vida del hombre contra la malicia del hombre" (2002:XIII).

${ }^{14}$ En cierta medida se puede decir que Gracián es uno de los primeros psicólogos en el sentido que Nietzsche le da al término. Su moral se basa en gran parte en la observación de la naturaleza humana y en prescribir reglas que nos permitan lograr la mayor utilidad. Quizás, puede ser de particular interés el que esta moral no sea de orden contemplativo (tal como sería de esperar en un religioso), sino que sean reglas de carácter mundano. Romera-Navarro nos dice al respecto: "Da él reglas para triunfar en el mundo. Algunas son egoístas y cautelosas, como el vivir práctico demanda; la mayoría son las propias de la moral prudencia. No se dirige a hombres contemplativos que viven alejados del ruido del mundo y pueden practicar cómodamente la virtud. Se dirige a criaturas de carne y hueso entregadas a la batalla de la existencia. Mira a la conveniencia no al sacrificio” (1935:121).

${ }^{15}$ García-López recoge algunos ejemplos: "Su opinión respecto del momento actual suele ser, en la mayor parte de los casos, desfavorable: "Lo antiguo... fue siempre lo mejor"; "van degenerando los hombres"; "no es siglo de hombres eminentes". No obstante, en otras ocasiones nos dirá que el pasado en nada difiere del presente. "Lo que sucedió doscientos años ha, eso mismo estamos viendo", pues el mundo "el mismo fue siempre que es”. La naturaleza una veces será “sabia” y “atenta” y otras “madrastra” del hombre e "imperfecta” (1947:28-29).
} 
uno de los fines de esta nueva forma de praxis. ${ }^{16}$ Además, el control sobre sí no sólo se restringe a limitar nuestras pasiones. Buena parte de él consiste en aplicarse a mudar nuestra disposición natural por medio del artificio, de lo contrario, el mejor hombre resulta ser tan sólo un hombre tosco (2002:XII). El ser humano, según Gracián ha de ocultar su interioridad. Pasiones e intenciones, no deben revelarse, salvo como un falso desliz, pues el jesuita está atento a que el ocultamiento constante es sospechoso y termina por traicionar su propósito, dando a conocer lo que se quería mantener en secreto. La conducta no debe ser previsible; ni siquiera el mismo acto de disimular. ${ }^{17}$ Todo esto nos podría sugerir que la actitud desconfiada ante el mundo y la visión, más bien pesimista del ser humano, decantarán en un juicio agrio frente a la apariencia, es decir, como una condena; sin embargo, el arte de fingir o de adornar es, sino alabado, al menos apreciado. Nada está libre de requerir un modo adecuado, no sólo se trata de "endulzar" la verdad, sino que esta práctica es requisito indispensable para que las cosas permanezcan. ${ }^{18}$

En relación con la felicidad, requisito indispensable es la sindéresis (buen sentido) que trata del fundamento de la prudencia y consiste en la capacidad de acertar en las situaciones con lo más adecuado (2002:XCVI). Sin embargo, Gracián se distancia de la tradicional noción de la aurea mediocritas; para él parte de la prudencia es no dejarse satisfacer con las grandes cosas sino quedar siempre presto a ir más allá (2002:CII). Pero, volviendo al ocultamiento, la propia buena ventura debe ser resguardada de ataques y de la envidia, siendo parte de la felicidad, precisamente, que no se muestre sino exhibir, de vez en cuando, alguna falla (2002:LXXXIII).

Si Gracián aunaba simulación y disimulación como dos instrumentos legítimos de la supervivencia y de éxitos mundanos, T. Accetto propone su separación, por medio de lo cual plantea validar la disimulación y condenar la

\footnotetext{
16 “Es el arte de mover voluntades; más consiste en destreza que en resolución: un saber por donde se le ha de entrar a cada uno. No hay voluntad sin especial afición, y diferentes según la variedad de los gustos. Todos son idólatras: unos de la estimación, otros del interés, y los más del deleite. La maña está en conocer estos ídolos para el motivar, conociéndole a cada uno su eficaz impulso: es como tener la llave del querer ajeno" (2002:XXVI).

17 "No siempre de un modo, para deslumbrar la atención, y más si émula. No siempre de primera intención, que le cogerán la uniformidad, previniéndole, y aun frustrándole las acciones. Fácil es de matar al vuelo al ave que le tiene seguido, no así la que le tuerce. Ni siempre de segunda intención, que le entenderán a dos veces la treta. Está a la espera la malicia; gran sutileza es menester para desmentirla. Nunca juega el tahúr la pieza que el contrario presume, y menos la que desea” (2002:XVII).

18 "No basta la sustancia, requiérese también la circunstancia. Todo lo gasta un mal modo, hasta la justicia y la razón. El bueno todo lo suple: dora el no, endulza la verdad y afeita la misma vejez" (2002:XIV).
} 


\section{Boris Eremiev Toro}

simulación. ${ }^{19}$ La disimulación permite salvar al hombre y resguardarlo; la simulación lo condena. Disimular es, en buena parte, un ejercicio de virtudes cuando su fin es la cautela. No obstante esta supuesta llaneza del discurso, las paradojas no faltan, pues Accetto parte excusándose de la brevedad de su tratado, prometiendo suplir más adelante su falta de calidad con una mayor cantidad de prosa para, luego, rematar su advertencia con la sorprendente confesión de que el escritor ha ido disminuyendo su obra. Esta disminución no ha sido sino efecto del propio tema a defender, a saber, la disimulación

Hace un año este tratado era tres veces más de lo que ahora se ve, y esto es conocido por muchos; y si yo hubiera querido demorar más en darlo a la imprenta, habría sido la vía de reducirlo en nada, por las continuas heridas que iban a destruirlo más que corregirlo. Se conocerán las cicatrices en cada buen juicio y seré excusado de presentar mi libro de este modo, casi exangüe, porque el escribir sobre la disimulación ha requerido que yo disimulara (2005:87).

En consecuencia, la disimulación se nos muestra como una práctica que, llevada al extremo, decanta en el ocultamiento, en la desaparición.

Este accionar lo coloca a Accetto en el mismo origen del hombre, pues recurriendo a Gen. 2.25, 3.7 y 3.10 hace notar que, surgido el pecado, se usó de la disimulación. Sin embargo, ésta no debe confundirse con la mentira, sino que el esfuerzo ha de dirigirse a distinguirla claramente. Para no caer en vicios ha de tenerse en cuenta la verdad: sólo con ella en mente podremos adentrarnos en el arte de fingir. A primera vista, no queda muy claro cuál es el propósito de este conocimiento, pues, podría bastar para disimular el saber ocultarse. Sin embargo, el interés de Accetto no sólo radica en entregar técnicas disimulatorias sino, también, en demostrar que ésta es justa y honesta. Así, Accetto nos explica muy sucintamente qué es la verdad. Lo primero es afirmar que verdad y bien no se distancian y que corresponde al bien que hay en las cosas. Después, que la verdad se efectúa en el intelecto y por último que es Dios la fuente y norma de la verdad misma. ${ }^{20} \mathrm{La}$ identificación entre el bien y la verdad, inmediatamente, nos lleva al problema de la legitimación de lo que no es verdad. Para salvar este escollo, Accetto distingue entre lo que es falso y el equívoco. Lo falso corresponde al ámbito del no ser, a su afirmación y antes de cometer semejante acto es preferible el

\footnotetext{
19 “Afirmo pues que mi fin ha sido tratar de cómo el vivir cauto bien se acompaña con la pureza del alma” (2005:85).

${ }^{20}$ A nuestro juicio, estos sucintos comentarios sobre la verdad sólo pueden entenderse dentro de la concepción tomista de la verdad, Cfr. especialmente De veritate 1.1. tomo 22 de la edición Leonina.
} 
silencio. En cambio, el equívoco —según Accetto- es un espacio entre nosotros y el mundo, espacio que nos permite un diferimiento de la verdad, una suerte de escudo ante los embates de la fortuna. ${ }^{21}$ Este espacio, debemos recalcar, no pertenece a nuestro ser propio, de lo contrario, no existiría esa auto-transparencia que nos es constitutiva; así no cabe en relación con nosotros el engaño. La prudencia corresponde a revelarse, por medio de dosis pequeñas o grandes según corresponda. Así, la honestidad del equívoco queda salvada; no es un engaño, un mostrar lo que no se es, sino un mostrarse a ratos. La simulación no goza de la misma estima para Accetto, pues, a diferencia de la disimulación ella implica el no ser, por lo cual, afirma confusamente el tratadista, conlleva la propia destrucción. ${ }^{22}$ Pero, la disimulación no queda entregada libremente a nuestro capricho. Ella obedece a ciertas limitaciones y fines. La justificación de la disimulación estriba en el no padecer daño, es un arma de defensa, no de ataque. Sólo podemos recurrir a ella cuando se nos presenta como el mal menor y, aún así, debemos usarla en contadas ocasiones pues, de lo contrario, ésta se podría volver en contra nuestra y, en lugar de protegernos por medio de nuestro ocultamiento, nos ganaría la fama de engañadores, con lo cual siempre se sabrían nuestras intenciones. Establecidos su propósito y sus límites, la disimulación nos exige conocer quiénes pueden disimular, cómo podemos lograrlo o, mejor dicho, qué requisitos se requiere para ser un buen disimulador; qué ventajas se obtiene con ella y frente a quiénes se puede disimular.

Los menos dotados para la disimulación son aquellos cuyo temperamento tiende a la transparencia y al descontrol. Así, el melancólico al deformar la realidad por sus estados de ánimo y al estar sujeto a un exceso de impresiones, tiende a revelarse. El alegre, en cambio, por su extrema vitalidad tiende a ser expansivo. El furioso, perdida la razón en su ira, no conoce el control y peca de ser claro en sus intenciones. El plácido tiende a mostrar tolerancia, pues no da valor a sus aflicciones. Sólo el temperamento moderado está bien dispuesto para el arte de la disimulación. Él es quien clausura sus emociones, no expresándolas en su rostro el que se vuelve un instrumento que usa a su antojo mostrando los sentimientos no según los padece, sino según le

\footnotetext{
21 "Pero como fuera del mundo se concede aquello que por los filósofos es denominado vacuum impropium, donde se recibiría la flecha lanzada por alguien que estuviera en la parte extrema del cielo, así el hombre, que es un pequeño mundo, a veces tiene fuera de sí cierto espacio que puede llamarse equívoco, no ya entendido como simple falsedad, a fin de recibir en él, por así decirlo, las saetas de la fortuna...” (2005:96).

22 "En el breve curso de los días, las horas o los momentos, como es la vida mortal, no sé por qué la propia vida se debería ocupar en destruirse más a sí misma, añadiendo la falsedad de las operaciones donde el ser casi no existe...” (2005:99).
} 


\section{Boris Eremiev Toro}

convenga. ${ }^{23}$ Aunque haya temperamentos más aptos para ocultarse, es posible adquirir este arte por medio del ejercicio. Para ello, es menester manejarse con dos registros de conocimientos: a saber, el que se adquiere por el conocimiento del mundo y sus costumbres y el que se adquiere por la lectura. Ambas fuentes confluyen en el conocimiento de los demás hombres con lo cual se puede tasarlos y saber actuar a sazón.

La disimulación no se reduce a una mera defensa sino que es, también, un valor positivo. Al menos así lo afirma Accetto, pues la belleza mundana — de carácter perecedero - basa su virtud en disimular su caducidad. Todo arte se reduce a no mostrar los defectos. Además, las buenas costumbres, es decir, el arte de saber convivir con los demás, es un desideratum del ocultamiento, pues se reduce a restringir los propios deseos y a ocultar el percatarse de los ajenos. A estas ventajas debemos agregar los placeres que implica el disimular. Aunque sea penoso el saberse guardar los propios pareceres - no en vano decimos en esta situación que hay que morderse la lengua - también es fuente de gozo, según Accetto, el "haber usado sobriedad de palabras y de acciones” (2005:117).

Frente a los demás, la disimulación es fácil cuando se trata de fuertes y de aquellos que muestran su poder de manera directa; no así frente a los astutos, pues si son buenos disimuladores no sabremos quienes son debiendo enfrentarlos en su mismo terreno. La astucia aquí es, precisamente, el no serlo a la vista de los demás; o, dicho de otro modo, es más astuto quien pasa por tonto.

Accetto también considera los obstáculos que existen a la hora de disimular. Dentro de ellos, la ira es uno de los más grandes. La ira, de entre todas las pasiones, es la más contraria al ocultamiento, pues expresa toda su intención y los propios conceptos, mutilados por la fuerza de la emoción, demuestran la perturbación interior, sin dejar de lado que el propio rostro se transfigura fácilmente por su causa. El poder de la ira radica especialmente en ser una mezcla de placer y de molestia. ${ }^{24}$ Para combatir este elemento positivo, debe considerarse el placer que implica el vencerse a uno mismo.

\footnotetext{
23 "El moderado es muy hábil para el efecto de la prudencia, porque ha de ser, en las tempestades del corazón, toda serena la cara o, cuando es tranquilo el ánimo, parecer turbado el rostro, según lo requiera la ocasión” (2005:105). El tema del rostro como lugar de manifestación de las pasiones es algo que es tematizado durante el s. XVII. Se trataba de revivir la tradición fisiognómica en función de la política, el buen político — cuyo paradigma es Richelieu - será aquel cuyo rostro y designios permanecen escindidos y que tenga a su vez la capacidad de leer el rostro ajeno (Bodei, 1995:141-143).

${ }^{24}$ Accetto parece basarse para hacer esta afirmación en Rhet. 1370b, donde se dice que: "Por lo cual es agradable el encolerizarse, como cantó Homero acerca de la ira: 'la cual es más dulce que la miel gota a gota destilada”. Pero es en Rhet. 1378a-1378b, donde Aristóteles trata con más detención el tema de la ira.
} 
Tener un concepto demasiado grande de sí, también constituye un gran impedimento para el disimular, pues, aquel que lo haga suele considerarse maestro de los demás, hablando en demasía de cosas que sería mejor callar. En cambio, el silencio es lo más adecuado, pues, es el resultado de la apreciación justa de sí y de no dar más de lo que se sabe. Asimismo, ser testigo de los éxitos de los ignorantes puede provocar sentimientos que atenten contra la disimulación, pero es suficiente consuelo considerar que los dones de la fortuna otorgados sin razón fácilmente perecen, mientras que los frutos del talento tienden a ser reconocidos, si no en esta época, al menos en otra posterior. Dentro de esta misma línea, nos advierte Accetto que frente a los poderosos lo mejor es disimular las venturas y las desventuras, no sólo de los bienes externos, sino también los del alma, pues esta precaución evita la envidia, sobre todo, la de aquellos que detentan el poder sin merecerlo. Quien no puede realizar estos actos y no es capaz de disimular las injurias está presto a buscar venganza. De este modo no sólo se expone a los riesgos que ella implica, sino que se ve privado de la tranquilidad de ánimo. La salud y la tranquilidad del hombre radican en la ocultación de nuestra intimidad. Este resguardo está en nuestro poder, por ende, es nuestra responsabilidad el conseguir nuestro bienestar.

La disimulación es una actividad ínsita a la vida terrena $\mathrm{y}$, en consecuencia, no podemos dejar de llevarla a cabo, mientras estemos en este mundo. Acceto, en efecto, considera que sólo en el día del Juicio la disimulación se volverá gratuita. En este tiempo, Dios juzgará de los corazones de los hombres y todo ocultamiento será vano.

\section{CONCLUSIÓN: FRAGMENTA HISTORIOLAE SIMULATIONIS}

Hemos procurado rastrear, brevemente, la paradójica historia de la di/simulación. Paradójica, pues en un primer momento surgió aparejada a un carácter problemático: ser un vicio más tolerable que su contrario la jactancia, para luego devenir en la más alta forma de la cautela y de la prudencia. Aristóteles es quien primero la condena y la arrincona, dejándole un pequeño respiro como una forma de humor propia de los caballeros. Cicerón parece tomarle la mano. También la considera un aderezo del discurso, una forma elegante de lo cómico que no tiene cabida en el mundo de la vida. Sin embargo, la situación se trastoca en el Barroco de Gracián y de Accetto cuando tanto la disimulación como la simulación tendrán su vindicación. La situación política obliga al hombre a replegarse sobre sí mismo y a dividir su interior y su exterior, procurando salvaguardar así su interioridad ante un mundo falaz y hostil. La disimulación se convertirá en un arma ofensiva y la disimulación en un medio de defensa. Ambas para Gracián son artificios 
necesarios para moverse en un mundo de apariencias donde la misma verdad ha de ser disimulada y simulada (dorada). Lo mundano es el mundo en el cual estos artificios y simulacros tienen cabida y toda dimensión trascendente parece suprimida para Gracián. Accetto basa la escisión entre simulación y disimulación en lo teológico. Disimulación y simulación son fruto de la caída, pero sólo la primera tiene un carácter honesto y puede utilizarse sin menoscabo de la salvación de nuestra alma. De este modo, ambas formas de la apariencia terminan adquiriendo carta de ciudadanía en el vivir humano.

Lejos de pretender que esta reseña del ocultamiento sea exhaustiva, creemos que es un acicate para continuar rastreando sus apariciones y $\dot{\mathrm{C} P o r}$ qué no, sus omisiones y silencios?

\author{
Pontificia Universidad Católica de Chile* \\ Facultad de Filosofía y Estética \\ Instituto de Filosofía, Campus San Joaquín \\ Avda. Vicuña Mackenna 4860, Macul. C.P. 7820436, Santiago (Chile) \\ beremiev@puc.cl
}

\title{
BIBLIOGRAFÍA
}

ACCETTO, T. La disimulación honesta. Buenos Aires: El cuenco de plata, 2005.

ARISTÓTELES. La Retórica. Buenos Aires: Eudeba, 2006.

------ Etica Nicomáquea. Etica Eudemia. Madrid: Gredos, 1985.

BODEI, R. Geometría de las pasiones. Ciudad de México: E. F. C., 1995.

CANTER, H. V. "Irony in the Orations of Cicero", en The American Journal of Philology, Vol. 57, N 4 (1936):457-464.

CICERÓN, M.T. Sobre el orador. Madrid: Gredos, 2002. Sobre los deberes. Madrid: Alianza, 1989.

GARCÍA-LÓPEZ, J. Baltasar Gracián. Barcelona: Labor, 1947.

GRACIÁN, B. Oráculo manual y arte de prudencia. Barcelona: Debate,2002.

HATZFELD, H. Estudios sobre el Barroco. Madrid: Gredos, 1966.

MARAVALL, J. A. La cultura del Barroco. Barcelona: Ariel, 1975.

PAVLOVKIS, Z. “Aristotle, Horace, and the Ironic Man”, en Classical Philology, Vol. 63, N 1 (1968):22-41.

ROMERA-NAVARRO, M. "Sobre la moral de Gracián”, en Hispanic Review, Vol. 3, N 2 (1935):119-126.

SANCTI, Thomae de Aquino. Opera omnia, Iussu Leonis XII P. M. Tomus XXII. Romae: AD Santae Sabinae, 1970. 